\title{
Research on Color Luminance Ratio Design of Architectural Accessible Signs
}

\author{
Jia Weiyang ${ }^{1, a}$ \\ ${ }^{1}$ School of Architecture, Tianjin University, No.92 Weijin Road, Nankai District, Tianjin, P.R.China \\ ajiaweiy@163.com
}

Keywords: accessible signs, color contrast, luminance ratio, sight distance, binocular visual acuity Abstract: Luminance ratio or contrast is a key indicator for accessible signs' color design. Existing researches had different results due to inadequate consideration of influencing factors. This project designed a subjective evaluation experiment within normal illumination environment, the minimum luminance ratio to identify an architectural accessible sign was statistically analyzed under different hue scheme, sight distance and visual acuity.

\section{Introduction}

The increasing elderly and the large number of disabled population have become severe social problems in China. At the end of 2015, the national population over 60 years old had exceeded 222 million, of which 144 million were over 65 years old ${ }^{[1]}$; before 2010 , the number of disabled people in China is about 85.02 million $^{[2]}$. The total of these two vulnerable groups had exceeded $21 \%$ of China's population. The construction and improvement of the barrier-free environment is an important guarantee for the life and travel of the elderly and the disabled. The accessible sign is an indispensable part and relatively easy to upgrade. At present, the design of accessible sign in China is still weak in scientific research. For example, the new Codes for accessible design of China had put forward the requirement of "eye-catching" and "strong contrast", but still lacked quantifiable and applicable reference indicators for color design.

\section{The relevant parameters of color design for accessible signs}

According to universal design theories, besides ordinary people, an accessible sign's color still need to meet the requirements of the elderly and visually impaired. The first principle to follow for accessible sign design is "easy to identify", and based on architectural physics, affecting factors include visual acuity, contrast, visual angle (Ratio between object size vertical to sightline and sight distance), illumination, recognition time, glare and so on. While luminance ratio $(L R)$ or contrast value is a key indicator directly related to color design; visual acuity, sight distance (SD), sign scale and illumination are also closer relevant factors. In current the vision impaired in China is defined as that the corrected visual acuity of better eye is less than $0.3 ; S D$ is also an important parameter of color design. Using binocular visual acuity (BVA) is more like the actual conditions in real life.

\section{Research review on color design for accessible signs}

The researches on signs for visually impaired persons are all currently based on subjective evaluation experiments. A study from Georgia Institute of Technology in 1985 showed that the minimum contrast for visually impaired to recognize easily is $70 \%{ }^{[3]}$, and was once written into the technical manual of Architectural Barriers Act; in 1995 Japanese scholar Yoshida Mai and her colleagues found that the minimum LR required is 1.5-2.0 (contrast 33-50\%) for the elderly ${ }^{[4]}$; an architectural interface study by Brett and Cook (2004) in U.K. for visual impairment required minimum contrast of $30 \%{ }^{[5]}$. Many scholars thought these indicators as poor scientificity due to the lack of adequate influence factors. for instance, Brabyn (1991) pointed out that the results were not applicable if no limits about minimum luminance ${ }^{[6]}$; a report by Volpe National Transportation Systems Center 
(1993) also demonstrated that the minimum color contrast correlated to the hue and $S D$, and signs are often with standard colors such as green \& white, blue \& white, black \& yellow, while human sensitivities were different for light colors, and their experiment shows that the yellow logo only required a contrast of $40 \%{ }^{[7]}$. Because of controversies, the Americans with Disabilities Act deleted the reference indicators in Architectural Barriers Act accessories. Thus sign color researches should analyze the missing impact factors, improve experiments and statistics, and put forward more reliable, easy-to-use results for architectural design.

\section{Subjective evaluation experiment scheme for accessible signs' color}

With good reading luminous environment (greater than 1001x according to China Codes) and vertical viewing angle, the correlation coefficient and regression relationship between $L R$ and $S D$ or $B V A$ were counted. Students with different $B V A$ were instructed to recognize the international standard barrier-free symbol (ISBFS) with 3 color schemes (green \& white, blue \& white, black \& yellow) and different $L R(1.12 \sim 12.03$ measured $)$, at medium and short $S D$ of $(0.6 \mathrm{~m}, 1 \mathrm{~m}, 2 \mathrm{~m}, 3 \mathrm{~m}, 4 \mathrm{~m}$, $5 \mathrm{~m})$, to get the minimum $L R$ value clearly identified.

The main instruments and materials are: color luminance meter, illuminometer, dual distance standard visual acuity chart (take the average of $B V A$ at $3 \mathrm{~m}$ and $5 \mathrm{~m}$ ), 3 color schemes of ISBFS samples (as Fig. 1, designed with different $L R$, size $200 \times 200 \mathrm{~mm}$ ), and the experimental data scale.

Experimental environment: standard visual acuity chart and each ISBFS sample in a fixed position, posted on the vertical whiteboard, with measured surface illumination of 3101x.

Testees: based on biostatistics sample requirements, 30 college students volunteer were invited involved in the naked eye test.

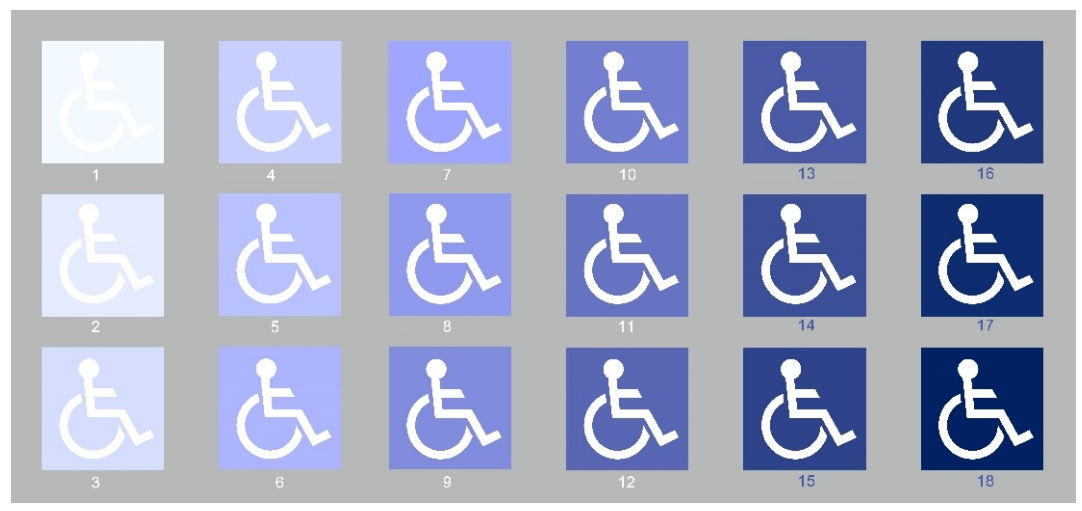

Fig. 1 signs in gradient LR (designed and drawn by the author)

\section{Statistical analysis of experimental data}

The experimental obtained a total of 540 valid data of 30 volunteers. Multiple sets of data regression mainly analyzed statistics of subjects whose $B V A$ were less than 0.4 . As a literature ${ }^{[8]}$ indicated, most people's $B V A$ are higher one line (about 0.1 ) than one eye. As the standard of visual disability in China is defined as that the visual acuity of better corrected eye is less than 0.3 , so $B V A$ less than 0.4 is approximately equal to the standard. There were 20 testees whose $B V A$ without correction were less than 0.4 .

\section{(1) The relationship between $S D$ and $L R$ in different color schemes}

The results (Table 1$)$ show that the $L R$ required at close distance $(0.6 \mathrm{~m}$ and $1 \mathrm{~m})$ for each color scheme come near; while at medium distance $(2 \mathrm{~m} \sim 6 \mathrm{~m})$, green $\&$ white color sign required the lowest $L R$, blue \& white followed, and black \& yellow required highest $L R$. This experimental result seems to be not consistent with the common sense of high sensitivity to yellow-green light for human eyes. That yellow \& black sign was hard to recognize could be interpreted as the main signage with deep color is less sensitive to human eyes, in other words, deep background with bright graphics is better to read 
than the opposite color scheme. It can be drew that a color scheme including white graphic and deep background is more identifiable, better with illumination and increasing illuminance appropriately.

Table 1

\begin{tabular}{llllllll}
\hline & $\mathbf{0 . 6 m}$ & $\mathbf{1 m}$ & $\mathbf{2 m}$ & $\mathbf{3 m}$ & $\mathbf{4 m}$ & $\mathbf{5 m}$ \\
\hline green \& white & 1.26 & 1.26 & 1.26 & 1.74 & 1.74 & 2.91 \\
blue \& white & 1.21 & 1.32 & 1.40 & 3.22 & 4.39 & 4.93 \\
black \& yellow & 1.23 & 1.23 & 1.83 & 6.67 & 10.20 & 10.35 \\
\hline
\end{tabular}

Note: The signs were all international standard barrier-free symbol, with size of $200 \mathrm{~mm} \times 200 \mathrm{~mm}$, surface illuminance $3101 \mathrm{x}$.

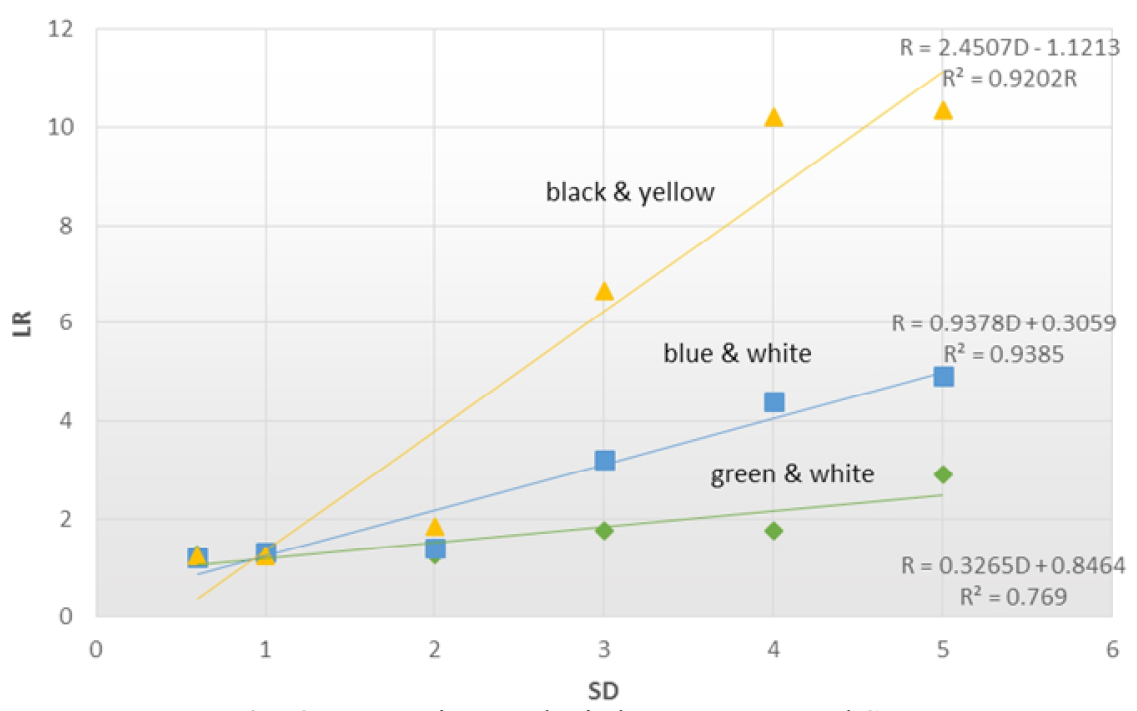

Fig. 2 Regression analysis between LR and SD

It also can be drew that in the medium and short $S D$ situation commonly used in architectural design, the longer the $S D$, the bigger $L R$ value required for signs to be recognizable. The regression analysis of the experimental data between the minimum $L R$ and $S D$ (Fig. 2) resulted in a better fitting degree $\left(\mathrm{R}^{2}\right)$. The results are as follows:

$\mathrm{R}_{\mathrm{L}}($ Green \& white $)=0.3265 \mathrm{D}+0.8464$

$\mathrm{R}_{\mathrm{L}}($ Blue \& white $)=0.9378 \mathrm{D}+0.3059$

$\mathrm{R}_{\mathrm{L}}($ Black \& yellow $)=2.4507 \mathrm{D}-1.1213$

Note: $\mathrm{R}_{\mathrm{L}}-$ the minimum $L R$ value recognizable, $\mathrm{D}-\operatorname{sight}$ distance $(\mathrm{m})$.

\section{(2) The relationship between $L R$ and $B V A$}

The regression analysis can be calculated form the data of tested students whose $B V A$ less than 0.4 approximate to visual impaired. Although the minimum $L R$ decreased along with the $B V A$ increased, yet there was no remarkable regression relationship (the $\mathrm{R}^{2}$ values were too low).

(3) The multiple regression analysis of $L R, B V A \& S D$

A multiple regression of the green and white scheme from 60 valid data for all the students were mounted as:

$\mathrm{R}_{\mathrm{L}}=1.2284-0.0782 \mathrm{~V}+0.0547 \mathrm{D}$

Note: $\mathrm{R}_{\mathrm{L}}$ - the minimum $\mathrm{LR}$ value recognizable, $\mathrm{V}-\mathrm{BVA}, \mathrm{D}$ - sight distance $(\mathrm{m})$. 
But its adjusted fitting degree is relatively low as 0.15 , indicating that reference value for scientific research or design practice of this formula is not high. At the same time, the correlation coefficient calculated between $L R$ and $S D$ is $0.301, L R$ and $B V A$ is -0.1809 . The result verified that the regression relationship between $L R$ and $B V A$ is not remarkable. In real design program, $S D$ is usually significant in line with the function and scale of the sign, but the user's diversity determines that we cannot limit the user's $B V A$, that is to say the $B V A$ parameter could usually be ignored when determining the color $L R$ value for an architectural sign.

\section{Conclusion}

Architectural accessible signs in medium and close sight distance with surface illumination conforming to the specification, should be designed with high contrast color scheme, better with white graphics and deep color background. In practice, the minimum $L R$ value between the graphics and background should be determined by $S D$ as the following Table 2 to ensure legibility:

Table 2

Refference value of LR \& color contrast (the author's statistics)

\begin{tabular}{lll}
\hline Sight distance & Refference LR & Color contrast \\
\hline$\leq 2 \mathrm{~m}$ & $\geq 2.0$ & $\geq 50 \%$ \\
$2 \mathrm{~m}-5 \mathrm{~m}$ & $\geq 5.0$ & $\geq 80 \%$ \\
\hline
\end{tabular}

China's specifications and codes lack quantitative standards for the accessible signs' color design, but the legibility of color is of great significance for promoting the quality and function of the accessible building environment. The research of this subject has put forward some color contrast and scheme design strategies for accessible signs, to provide reference for standard revision and design practice, with better scientificity and practicality, in order to improve the living environment for the elderly, the disabled and the whole society.

\section{Acknowledgements}

This work was financially supported by Natural Science Foundation of China (No. 51408404) \& Application Foundation and Frontier Technology Research Program of Tianjin (15JCYBJC22200).

\section{Literature References}

[1] Information on http://www.mca.gov.cn/article/zwgk/mzyw/201607/20160700001136.shtml.

[2] Information on http://www.cdpf.org.cn/sytj/content/2012-06/26/content_30399867.htm.

[3] Georgia Institute of technology. Signage for low vision and blind persons:A multimedisciplinary assessment of the state of the art. ATBCB,1985.

[4] Yoshida Mai. Changes of the elderly's yellow visibility (2) Luminance ratio analysis of interior finishing material color. Summary Proceedings of Japan Architecture Academy E-1 Architecture \& Plan Volume 1: 763-764.

[5] BRIGHT, K. T. and COOK, G. K. Project Rainbow, a research project to provide colour and contrast design guidance for internal built environments. Occasional Paper 57. ascot: the Chartered institute of Building, 1999.

[6] Brabyn, J., Co-director. Rehabilitation Enginerring Center, the Smith-Kettlewell Research Institute. Letter dated March 11,1991, to the office of the General Counsel,ATBCB.

[7] Bentzen, B. L., T. L. Nolin, R. D. Easton, and L. Desmarais. Detectable warning surfaces: Detectable by individuals with visual impairments, and safety and negotiability for individuals with physical impairment. Volpe National Transportation Systems Center:1993.

[8] Zhou Xiaodong, Wang Fangrun. Comparison of binocular and monocular visual acuity. Chinese School Health,1999,20(1):69 